\title{
Modeling and Characteristic Analysis of Wireless Ultrasonic Vibration Energy Transmission Channels through Planar and Curved Metal Barriers
}

\author{
DingXin Yang $\mathbb{D}^{\mathbb{D}}$, BaoJian Hou, Dong Tian, and Siyuan Wang \\ Laboratory of Science and Technology on Integrated Logistics Support, National University of Defense Technology, \\ Changsha 410073, China
}

Correspondence should be addressed to DingXin Yang; yangdingxincn@163.com

Received 25 August 2017; Revised 18 December 2017; Accepted 27 December 2017; Published 24 January 2018

Academic Editor: Filippo Ubertini

Copyright (C) 2018 DingXin Yang et al. This is an open access article distributed under the Creative Commons Attribution License, which permits unrestricted use, distribution, and reproduction in any medium, provided the original work is properly cited.

\begin{abstract}
Wireless ultrasonic vibration energy transmission systems through metal barriers based on piezoelectric transducers have drawn a lot of focus due to the advantage of nonpenetration of the barriers, thus maintaining the integrity of sealed structures. It is meaningful to investigate appropriate modeling methods and to characterize such wireless ultrasonic energy transmission channels with different geometric shapes. In this paper, equivalent circuit modeling and finite element modeling methods are applied to the planar metal barrier channel, and a 3-dimensional finite element modeling method is applied to the cylindrical metallic barrier channel. Meanwhile, the experimental setup is established and measurements are carried out to validate the effectiveness of the corresponding modeling methods. The results show that Leach's equivalent circuit modeling method and finite element modeling method are nearly similarly effective in characterizing the planar metal barrier channel. But for a cylindrical metal barrier, only the three-dimensional finite element modeling method is effective. Furthermore, we found that, for the planar barrier, the effect of standing waves on the efficiency of wireless energy transmission is dominated. But for the curved barrier, only the resonant phenomenon of the piezoelectric transducer exists.
\end{abstract}

\section{Introduction}

Traditionally, powering, communicating with sensors or electronic systems located in the isolated or hermitical metallic structures, requires the use of physical penetrations and wire feed-through. However, this approach has the disadvantage of limiting or potentially compromising the structural integrity and environmental isolation. Traditional electromagnetic approaches for wireless energy and signal transmission are inhibited in these applications due to the strong Faraday shielding effect presented by the metal barriers. As an alternative method, ultrasonic waves can be used to transmit vibration energy through a metal barrier wirelessly to power and communicate with the electronics enclosed in hermitical metal structures.

Such systems or acoustic-electric channels are usually formed by coaxially aligning and acoustically coupling a pair of piezoelectric transducers (PZTs) to opposite sides of a metal barrier. The outside transmitting piezoelectric transducer generates ultrasonic mechanical vibrations, and ultrasonic vibrations propagate through the metal barrier and are received by the inside receiving transducer and converted to electric energy.

It is of great importance to model the acoustic-electric channels properly and analyze the corresponding characteristics including the input impedance, the output-to-input voltage transfer ratio, and the energy transmission efficiency. All these characteristics are essential for optimal design of the channel. Methods for modeling the acoustic-electric channel mainly include theoretical analysis, equivalent circuit methods, and finite element modeling methods. Hu et al. [1$3]$ use two piezoelectric plates to form a sound power transformer and they have established the mathematical model of the system according to the acoustic wave equation and the linear piezoelectric equation. But only numerical simulation results are presented, they did not carry out experimental 
verification. Furthermore, the analytical model is difficult to incorporate the head, tail mass, and the coupling layer and to account for loss in all the mechanisms. Sherrit et al. [4] have introduced a kind of Mason's equivalent circuit network model in consideration of the effects of the back lining (air), and the energy transmission efficiency and other characteristics of the channel were obtained. Chang et al. [5] have also established the finite element model (FEM) of an energy transmission system and calculated the acoustic energy loss caused by the Lamb wave. Moss et al. $[6,7]$ have presented an equivalent circuit model of the sandwiched plate and developed an "acoustic-electric feed-thru" to transmit both power and data through the aluminum skin of aircraft using ultrasound. Wilt et al. [8-10] have also investigated finite element modeling for a two-transducer through-wall ultrasonic communication system and proposed one-dimensional pressure transfer models for the acoustic-electric transmission channels. Yang et al. [11] recently have modeled the acoustic-electric channel and analyzed the effect of load on transmission efficiency. However equivalent circuit method and FEM method mentioned above are mainly aimed at modeling and characterizing the acoustic-electric channel through planar metal barriers. Few researches are performed on channels through curved metal barriers. Furthermore, the adaptability of the modeling methods for wireless ultrasonic energy transmission channels through metal barriers with different geometric shape is rarely discussed.

In this paper, Leach's equivalent circuit model is established for the wireless ultrasonic energy transmission channel through a planar metal barrier. Energy transmission characteristics of the channel are analyzed in PSpice software based on the model. By comparison, a two-dimensional finite element model is also established for the same channel and simulation results are obtained using Comsol software. Both modeling results are compared with experimental measuring results to validate the effectiveness of the modeling methods based on the established physical channel and experimental setup. For a cylindrical metal barrier, the FEM method is the only suitable method to describe the channel. A kind of three-dimensional finite element model is built up and is used to analyze the characteristics of the channel which are compared with results from experimental measurements. Particularly, simulation and experimental results show that there are no multiple peaks and valleys in characteristics curves versus frequency for the cylindrical barrier channel which is different from what occurs in the planar metal barrier channel.

\section{Model of the Channel through a Planar Metal Barrier}

2.1. The Ultrasonic Vibration Energy Transmission Channel through a Planar Metal Barrier. The wireless ultrasonic energy transmission channel through a planar metal barrier under study is a sandwiched plate configuration, where two piezoelectric transducers are coupled directly opposite each other on a flat elastic slab of metal barrier coaxially using epoxy. As presented in Figure 1, a high frequency alternating power source ( $5 \mathrm{~V}$ peak sinusoidal) is applied to the electrodes of the transmitting transducer, and a load resistor $(R=$ $50 \Omega$ ) is connected across the output electrodes of the receiving transducer on the receiving end.

2.2. Leach's Equivalent Circuit Model of the Channel through a Planar Metal Barrier. Before establishing the whole acousticelectric channel's Leach's equivalent circuit model, we should establish Leach equivalent circuit of a piezoelectric transducer first. Leach's equivalent circuit model for a PZT is presented in Figure 2 which can be simulated in SPICE circuit simulation software [12].

In Figure 2, $\mathbf{F}_{\mathbf{1}}$ and $\mathbf{F}_{\mathbf{2}}$ represent the voltage-controlled voltage sources and $\mathbf{E}_{1}$ represents the current-controlled current source. Leach's piezoelectric transducer model has three ports: two mechanical ports denoted by $\mathbf{B}$ and $\mathbf{F}$ which represent the back and front of a piezoelectric transducer and a single electrical port $\mathbf{E}$ with the assumption that the opposite side of the piezoelectric transducer is grounded. The clamped capacitance $C_{0}$ is defined as

$$
C_{0}=\frac{\varepsilon_{33}^{S} A}{l_{p}},
$$

where $\varepsilon_{33}^{S}$ is the clamped permittivity of the piezoelectric crystal along thickness direction and $A$ is the cross-sectional area of the transducer's face. $l_{p}$ is the thickness of the transducer and $h_{33}$ is defined as

$$
h_{33}=\frac{e_{33}}{\varepsilon_{33}^{S}},
$$

where $e_{33}$ is the piezoelectric stress constant of the piezoelectric crystal along the thickness direction. $\mathbf{T}_{\mathbf{1}}$ presents the schematic of a lumped representation of an infinitesimally short segment of a distributed lossy transmission line which has in general the following distributed properties: inductance per unit length, $L$, capacitance per unit length, $C$, resistance per unit length, $R$, and conductance per unit length, $G$, respectively, which are defined as

$$
\begin{aligned}
& L=A \rho, \\
& C=\frac{1}{A \rho c^{2}}, \\
& R=\frac{\omega L}{Q_{m}},
\end{aligned}
$$

where $\omega$ is excitation angular frequency, and variables $\rho, Q_{m}$, and $c$ are the mass density, mechanical quality factor, and speed of sound in the piezoelectric transducer, respectively. Because the loss due to thermal conduction is negligible, conductance $G$ usually takes the value of 0 . While usually $R_{1}$ takes value of $1 \mathrm{k} \Omega$ and $C_{1}$ takes $1 \mathrm{~F}[12,13]$.

The propagation of mechanical waves in acoustic layers is analogous to the propagation of electrical waves in lossy transmission lines. We can use lossy transmission lines to represent the metal barrier and the coupling layers. Suppose that the PZTs are coupled to the metal wall using epoxy; there 


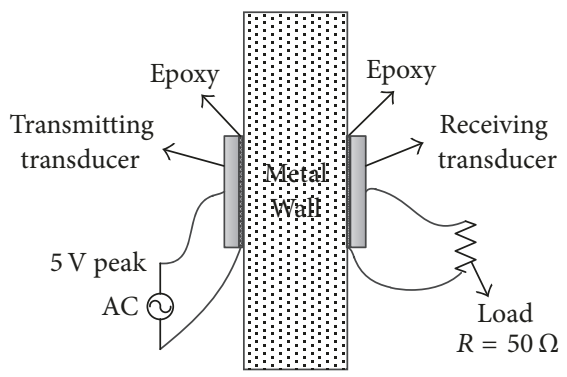

FIGURE 1: Diagram of the ultrasonic energy transmission channel formed by a pair of PZTs through a planar metal barrier.

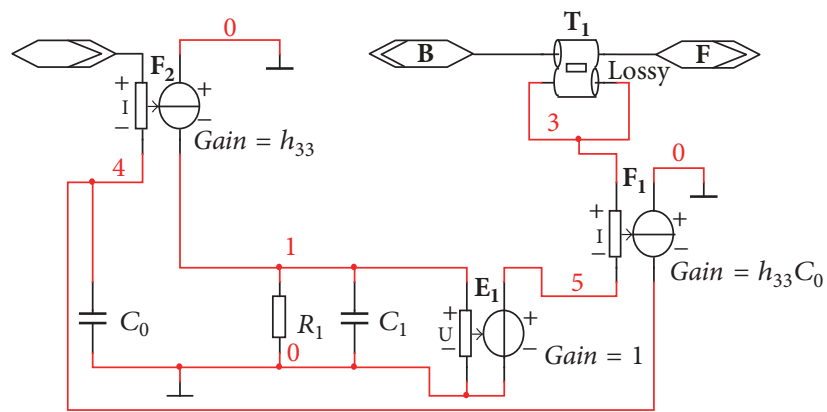

FIGURE 2: Leach's equivalent circuit model for a piezoelectric transducer.

are epoxy-wall-epoxy acoustic layers between the transmitting and receiving PZTs. These cascaded acoustic layers can be represented as a set of lossy transmission lines connected in series, where one lossy transmission line represents each acoustic layer. The lossy transmission line equivalent circuit model of three cascaded acoustic layers is shown in Figure 3.

The calculation of the lossy transmission line parameters for the metal barrier is the same as that of the piezoelectric transducer. For epoxy layer, the calculation of the corresponding lossy transmission line parameters $L, C$, and $G$ is the same as that of the piezoelectric transducer. But it is different for $R . R$ is calculated according to the equation of $R=2 \rho c A \alpha$, where $\alpha$ is the coefficient of attenuation due to viscous losses of the epoxy layer.

Then we can construct Leach's equivalent circuit model of the ultrasonic vibration energy transmission channel as shown in Figure 4. We can simulate and analyze the characteristics of the channel model in SPICE software environment. For verifying the models, the physical channel is constructed. The material of the transmitting and receiving piezoelectric transducers is PIC155. The transducer's resonance frequency is $2.009 \mathrm{MHz}$. The material properties and geometric parameters of the piezoelectric transducer are listed in Table 1, and material properties of the stainless steel and epoxy layers are listed in Table 2.

2.3. Two-Dimensional Finite Element Model for the Channel through a Planar Metal Barrier. For comparison with Leach's equivalent circuit model, the finite element model based on Comsol software is also established to characterize the channel through a planar metal wall. Comsol has the capability of combining multiphysics model with models of discrete electronic components. A two-dimensional finite element asymmetric model for the same channel as in Section 2.2 is shown in Figure 5. An alternative electric source $(5 \mathrm{~V})$ is applied to boundary (3), and boundary (2) is grounded. A pure resistive load is connected to the boundaries (4) and (5). Boundary (1) is the axis of symmetry.

The stainless steel and the epoxy are defined as the sound field model and the contact surface of the epoxy and the transducer is defined as the sound solid coupling contact surface, and the other boundaries are defined as the hard sound field boundaries. The closed circuit elastic stiffness of PIC155 $c_{33}^{E}=$ $8.85426 \times 10^{10} \mathrm{~N} / \mathrm{m}^{2}$. Then the grid is divided and solution is performed in the frequency domain. The equivalent input impedance and the output-to-input voltage transfer ratio of the channel can be obtained by calculations performed in Comsol.

\section{Three-Dimensional Finite Element Model for the Channel through a Cylindrical Metal Barrier}

The PSpice equivalent circuit modeling method mentioned above is applicable to the case where the metal barrier is planar and the ratio of the diameter of the piezoelectric ultrasonic transducer to the thickness is greater than 10 [6]. But when the metal barrier is curved, it is difficult to use the equivalent circuit model to characterize the acoustic-electric channel. The reason is that the curved metal barrier and coupling layers cannot be represented by using the lossy transmission lines like a planar barrier. For modeling channels 


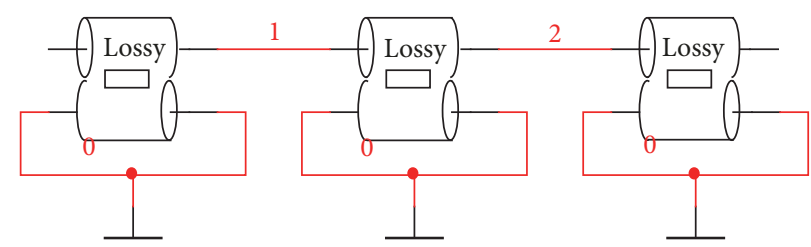

FIGURE 3: Equivalent circuit model of three cascaded dissipative acoustic layers.

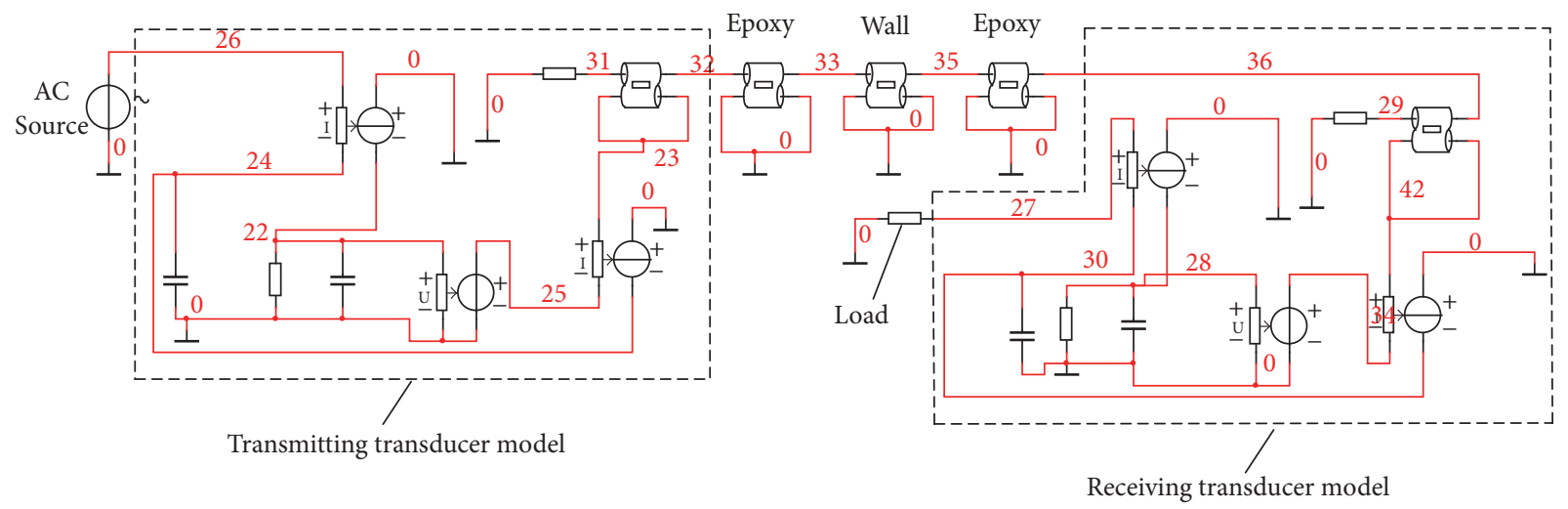

FIGURE 4: Leach's equivalent circuit model of the ultrasonic vibration transmission channel through a planar metal barrier.

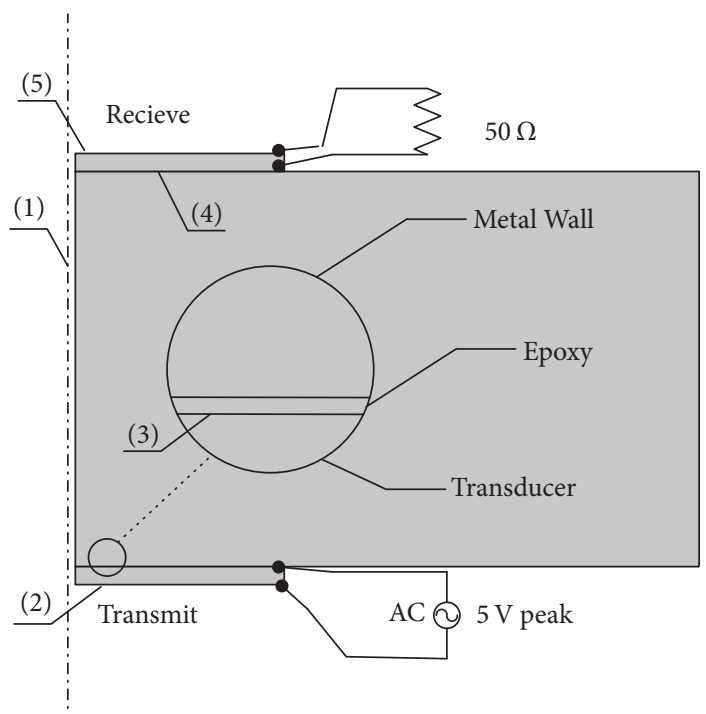

FIgURE 5: Two-dimensional finite element model for the ultrasonic energy transmission channel through a planar metal barrier.

TABLE 1: Material properties and geometric parameters of PIC155 piezoelectric transducer.

\begin{tabular}{lcccccccc}
\hline Transducer & $d(\mathrm{~mm})$ & $l_{p}(\mathrm{~mm})$ & $\rho\left(\mathrm{kg} / \mathrm{m}^{3}\right)$ & $h_{33}(\mathrm{~V} / \mathrm{m})$ & $\varepsilon_{33}^{S}(\mathrm{~F} / \mathrm{m})$ & $e_{33}\left(\mathrm{C} / \mathrm{m}^{2}\right)$ & $Q_{m}$ & $c(\mathrm{~m} / \mathrm{s})$ \\
\hline PIC155 & 20 & 1 & 7800 & $1.95 \times 10^{9}$ & $6.726 \times 10^{-9}$ & 13.12 & 80 & 3772 \\
\hline
\end{tabular}

TABLE 2: Material properties and geometric dimensions of other parts of the channel.

\begin{tabular}{lccccc}
\hline Material & $l_{p}(\mathrm{~mm})$ & $\rho\left(\mathrm{kg} / \mathrm{m}^{3}\right)$ & $\alpha$ & $Q_{m}$ & $c(\mathrm{~m} / \mathrm{s})$ \\
\hline Stainless steel & 22 & 7850 & - & 100 & 5790 \\
Epoxy & 0.032 & 1160 & 1.62 & - & 2620 \\
\hline
\end{tabular}


through metal barrier with complex geometric shape, FEM modeling method has its peculiar advantages, which can be applicable to metal barriers with an arbitrary geometric shape. Here a kind of three-dimensional finite element model based on Comsol is used for modeling and characterizing the ultrasonic power transmission channel through a cylindrical metal barrier.

In the channel, a pair of piezoelectric transducers is aligned concentrically on the inside and outside of a cylindrical metal barrier by epoxy. Because the transducer in this study is a planar disc with the same size as used in the planar metal barrier channel, it is necessary to add a round thin transition layer between the transducer and the cylindrical metal barrier, and the thinnest thickness of the transition layers is $2 \mathrm{~mm}$; the diameter of the transition piece is the same as the transducer. The material of the pipe and the transition piece is stainless steel. The pipe's inner diameter is $75 \mathrm{~mm}$ and external diameter is $85 \mathrm{~mm}$. The length of the pipe is $200 \mathrm{~mm}$ and the transducers are attached to the middle position of the length of the cylindrical steel pipe. The image of the constructed physical ultrasonic vibration energy transmission channel through a cylindrical steel pipe is shown in Figure 6.

As shown in Figure 7, a three-dimensional geometric model of the channel formed by the cylindrical steel pipe, transition pieces, and PZTs is established in SolidWorks. The geometric model can be imported into Comsol for performing further calculation and analysis.

In FEM simulation, an alternative source with voltage of $5 \mathrm{~V}$ is applied to Face 1 of the transmitting transducer as the electric excitation, and the opposite face of the transducer is grounded. A pure resistive load $(50 \Omega)$ is connected between Face 2 and the opposite face of the receiving transducer. In Comsol, the stainless steel pipe, transition piece, and the epoxy are defined as the sound field model and the contact surface of the epoxy and the transducer is defined as the sound solid coupling contact surface, and the other boundaries are defined as the hard sound field boundaries. After the dividing the calculation grids, solution can be performed in the frequency domain. Characteristics of the channel like the output-to-input voltage transfer ratio and equivalent input impedance can be obtained after a great amount of calculations.

\section{Experimental Setup and Modeling Methods Verifications}

To evaluate the correctness of the modeling methods mentioned above, we have established experimental setups to measure the equivalent input impedance and output-input voltage transfer ratio of physical channels through a planar steel wall and through a cylindrical steel pipe with the same parameters described in the foregoing sections, respectively. Experimental measurements are also carried out to validate these modeling methods by comparison with the simulation results from the models.

4.1. Equivalent Input Impedance Measurement of the AcousticElectric Channel. The illustration of the experimental setup for measuring the equivalent input impedance of the channel is shown in Figure 8. The external electrodes of a high precision impedance analyzer (Agilent 4294A) are connected with the electric terminals of the channel transmitting transducer. The receiving transducer is terminated with a load or is let open. Then the curve of the equivalent input impedance of the channel versus frequency is measured over the frequency range 1-3 MHz using Agilent 4294A. It should be pointed out that though the barrier in Figure 8 is a planar metal wall, the setup is also applicable to a cylindrical metal pipe.

4.2. Output-to-Input Voltage Transfer Ratio Measurement of the Acoustic-Electric Channel. The experimental setup illustration for measuring the output-to-input voltage transfer ratio the channel is shown in Figure 9. The transmitting transducer is connected with a signal generator AFG3021B which is connected with the computer through an USB interface. A frequency sweeping computer program is written to control the signal generator to produce sinusoidal voltages of different frequencies which are applied to input ends of the transmitting transducer. A multichannel digital oscilloscope TDS2012B connected with the computer through USB is used to record the input and output voltages. Sampling programs fulfill reading voltage waveforms into the computer from the digital oscilloscope. The input and output voltage waveform amplitudes are extracted from the sampling data, and voltage ratios at different frequencies are obtained.

The image of the experimental setup and the physical ultrasonic vibration energy transmission channel through a planar stainless steel wall is shown in Figure 10.

The measured output and input voltage waveforms are shown in Figure 11. It should be pointed out that the measured waveforms are composed of the discrete points with noise and the maximum value detection algorithm for amplitude calculation is not suitable for this case. We use the following integral calculation method to calculate the waveform amplitude.

$$
A=\sqrt{\frac{2}{T} \int_{0}^{T}[A \sin (\omega t+\varphi)+n(t)]^{2} d t},
$$

where $A$ is the wave amplitude, $T$ is the waveform period, $\omega$ is the angular frequency, $\varphi$ is the waveform phase, and $n(t)$ represents the measured noise.

4.3. Modeling Methods Verifications with Experimental Measurements. For the channel through a planar metal barrier, the characteristics from measurements are compared with that from equivalent circuit model and 2-dimensional finite model. Figure 12 shows the channel input impedance modulus and phase curves from three cases. It can be observed that the impedance curve from Leach's model is well matched to the curve from two-dimensional finite element model, which has multiple peaks and valleys. The results of the simulated curves are also nearly matched to the measured one throughout the frequency range except for some small deviations. The deviations may be due to the noise interference, the influence of epoxy coupling layer, and nonperfect flatness of the metal barrier. It can be observed that the two kinds of modeling methods can reproduce the multiple peaks 

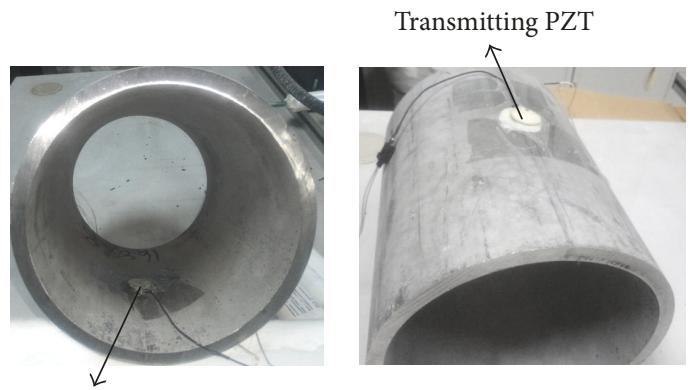

Receiving PZT

FIgURE 6: The physical ultrasonic vibration energy transmission channel through a cylindrical metal pipe.
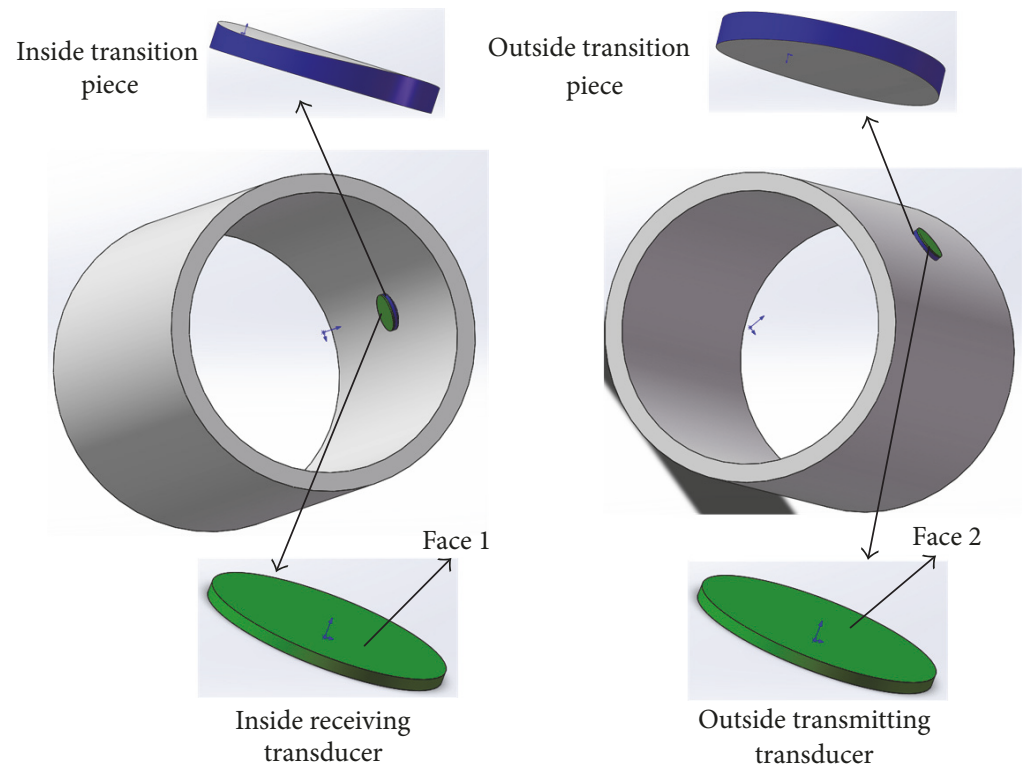

FIGURE 7: Three-dimensional geometric model of the wireless ultrasonic energy transmission channel through a cylindrical metal pipe.

and valleys appearing in the impedance versus frequency curves with a certain frequency interval. Because standing waves are formed in the planar metal barrier at some certain frequencies when incident acoustic waves constructively interact with the reflecting waves. Figure 13 shows the acoustic pressure distribution in the planar metal barrier from FEM simulations. We can see that there is a standing wave at the frequency of $2.009 \mathrm{MHz}$ as shown in Figure 13(a), none at the frequency of 2.24 MHz in Figure 13(b).

The output-input voltage transfer ratio curves versus frequency from Leach's equivalent circuit model, FEM simulation, and experimental measurements are shown in Figure 14. We can see that there is a good correlation between the three curves, and the shape of these curves is very similar to the shape of the curve which is obtained by the analytical method proposed by $\mathrm{Hu}$ et al. [1]. The three curves also have multiple peaks and valleys which are caused by the effects of the standing waves existing in the planar metal barrier. However there are some deviations of the positions of the peaks and valleys which may be due to the parameters measurement errors of the PZT and acoustic material, imperfect coupling between epoxy and the metal barrier, the noise interference, and so forth. It can be observed that there is a maximum voltage transfer ratio at the frequency of $2.263 \mathrm{MHz}$ which is more than 1 . We can use the simulated curves to find the best excitation frequency to achieve the maximum voltage transfer ratio.

From the above results we can draw the conclusion that the two modeling methods are suitable for characterize the acoustic-electric channel through a planar metal barrier and can be applied to the optimal design and performance prediction of the physical through-metal-wall ultrasonic energy transmission system.

For the channel through a cylindrical metal pipe, the equivalent input impedance modulus and phase curves versus frequency from Comsol finite element simulation and experimental measurement are illustrated in Figure 15. We can see that the impedance curve from finite element simulation is nearly matched to that from measurement except for a bit of frequency shift which may be caused by the geometric error of the actual channel, coupling effect, and so on. Another thing noticeable is that there are no multiple peaks and valleys in both simulation and experimental measurement results which is different from the phenomenon 


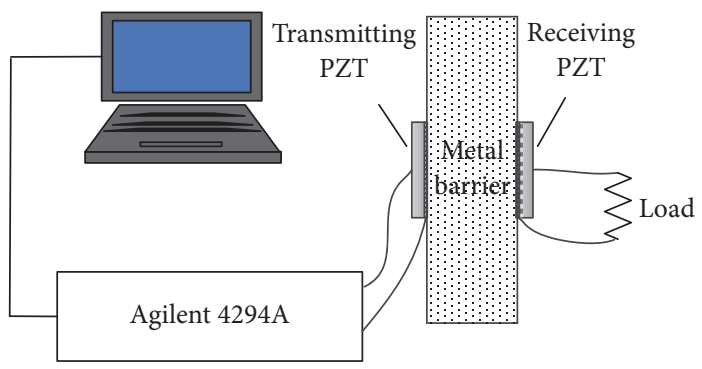

FIGURE 8: Experimental setup for measuring the input impedance of an acoustic-electric channel.

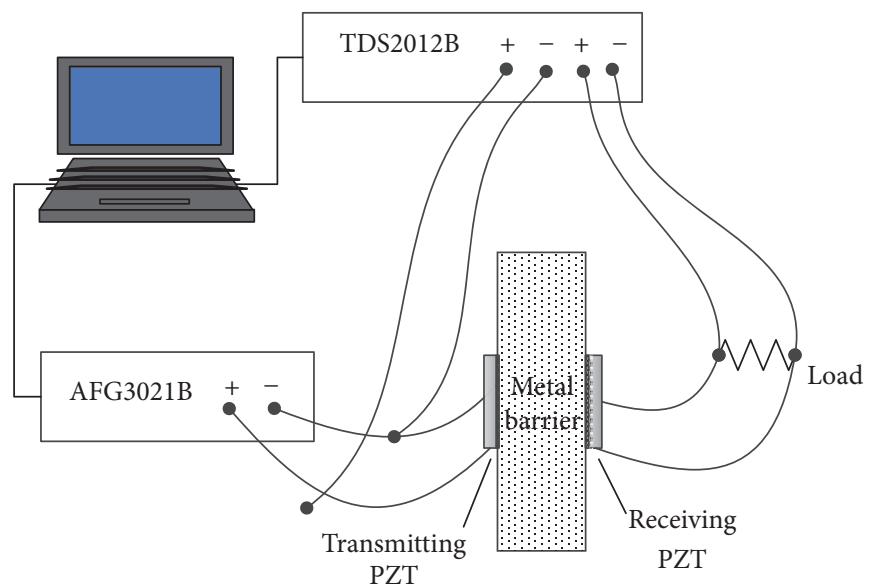

FIGURE 9: Scheme of the output-to-input voltage transfer ratio measurement of the channel.

occurring in impedance curves of the channel through a planar metal barrier.

Figure 16 shows the output-input voltage transfer ratio curves of the channel from Comsol finite element simulation and experimental measurement. There are some deviations between the measured and simulated results in the frequency range of 1.2-1.5 MHz. After $1.5 \mathrm{MHz}$, there is a bit better correlation. Both curves show the maximum voltage transfer frequency is near at $2.3 \mathrm{MHz}$. The voltage transfer ratio through the cylindrical metal wall is small and the measured maximum ratio is less than 0.05 , which means that it is more difficult for energy transmission through a cylindrical metal wall than through a planar wall. To compare with the standing waves occurring in a planar metal barrier, the acoustic pressure distribution in the cylindrical pipe is also calculated at the at the resonance frequency of $2.009 \mathrm{MHz}$ shown in Figure 17(a) and at the antiresonance frequency of $2.24 \mathrm{MHz}$ shown in Figure 17(b). It is obvious that we cannot see any standing waves existing in the cylindrical metal barrier. Nevertheless, the 3-dimensional finite model is shown to have the potentiality in modeling the wireless ultrasonic power transmission system through a cylindrical metal barrier with certain accuracy.

\section{Conclusion}

Wireless ultrasonic vibration energy transmission through metal barriers based on piezoelectric transducers has the advantage of maintaining structural integrity without penetration of the barriers. Appropriate modeling and characterizing such acoustic-electric channels through metal barriers with different geometric shapes are of great importance for optimal system design and performance prediction. In the paper, we use Leach's equivalent circuit model and twodimensional finite element model to characterize the energy transmission channel through a planar metal barrier. For the channel through a cylindrical metal barrier, it is difficult to characterize the channel's behavior using Leach's equivalent circuit model. A kind of three-dimensional finite element modeling method is adopted instead. In order to evaluate the correctness of modeling methods, the experimental setup has been established and experimental measurements are carried out to compare with channel characteristics from model simulations.

For the channel through a planar metal barrier, we compared the characteristics from measurement with that from equivalent circuit model and 2-dimensional finite model. Results have shown that Leach's equivalent circuit modeling method and two-dimensional finite element modeling method are nearly similarly effective in characterizing the planar metal barrier channel. Simulation curves are also nicely matched to the measured characteristics throughout the frequency range except for some small deviations. Standing waves are formed in the planar barrier which has been proved by both models simulations and experimental measurements. So equivalent modeling method and finite 


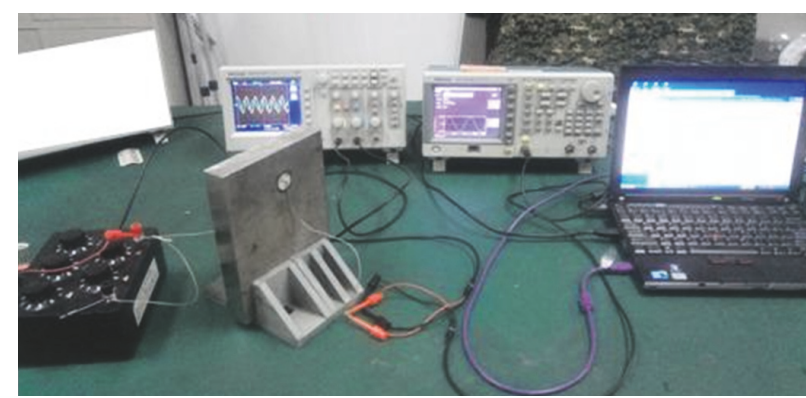

FIGURE 10: The physical acoustic-electric channel through a planar metal wall and experimental measurement setup.

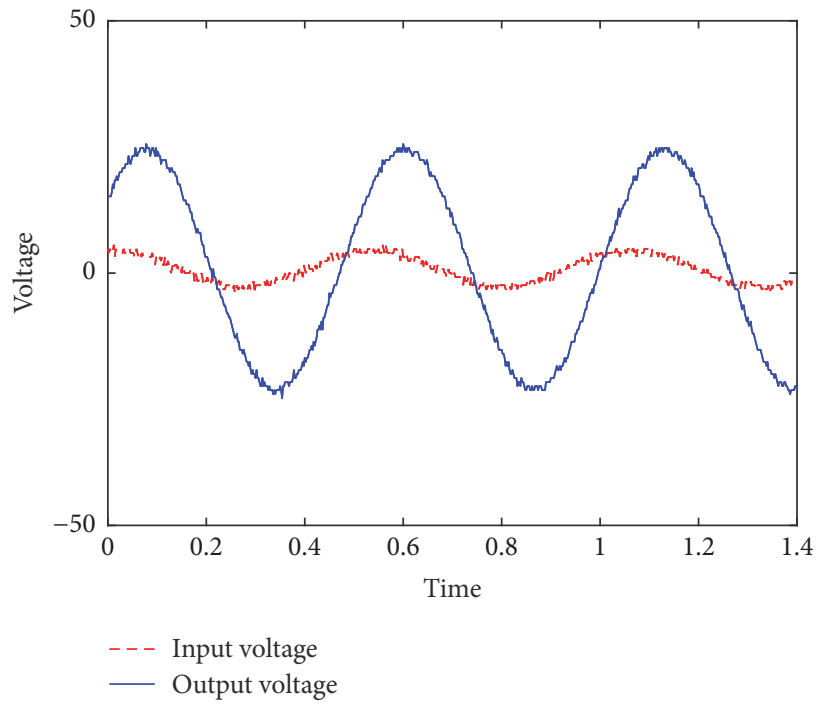

FIGURE 11: Measured input and output voltage waveforms.
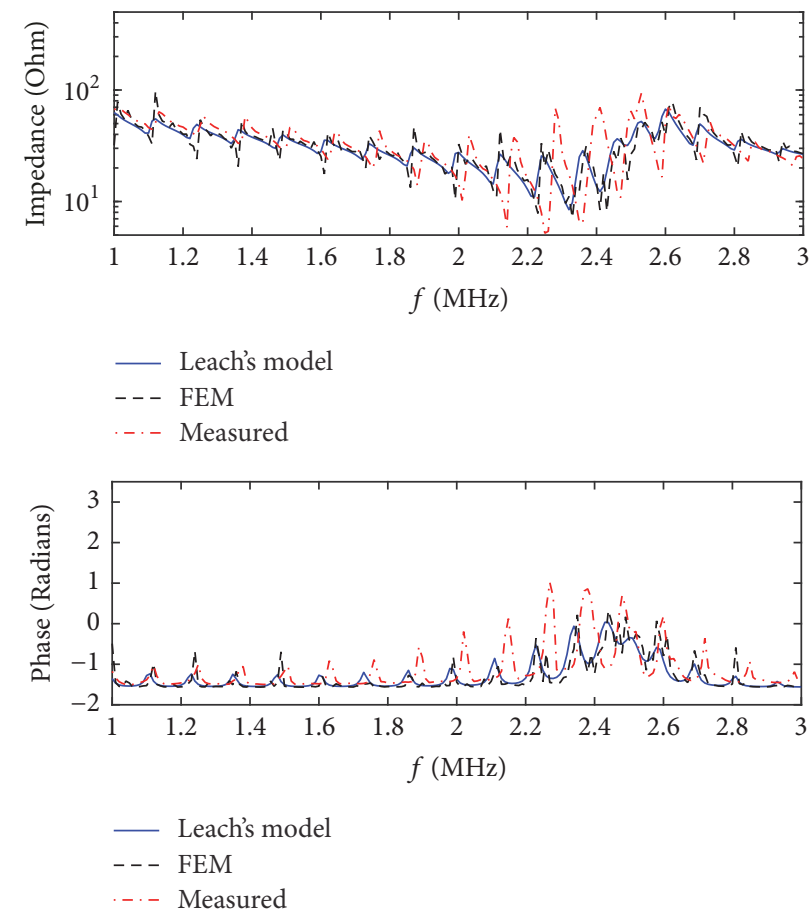

FIGURE 12: Equivalent input impedance of the channel through a planar metal wall. 


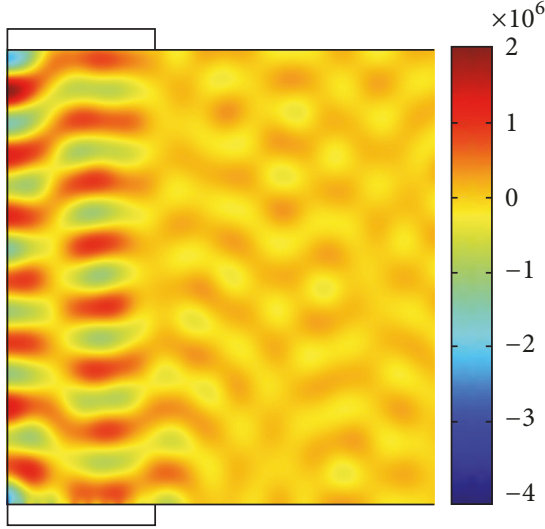

(a) $f=2.009 \mathrm{MHz}$

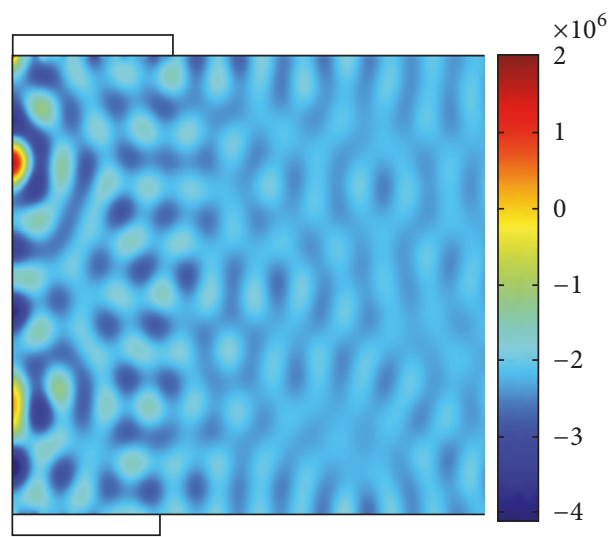

(b) $f=2.24 \mathrm{MHz}$

FIGURE 13: Acoustic pressure distributions in the planar metal wall from FEM simulations.

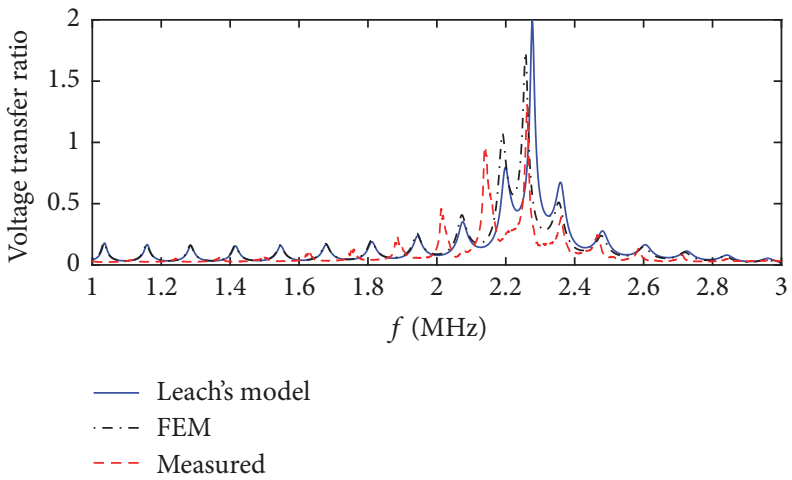

FIGURE 14: Output-to-input voltage transfer ratio of the channel through a planar metal barrier.
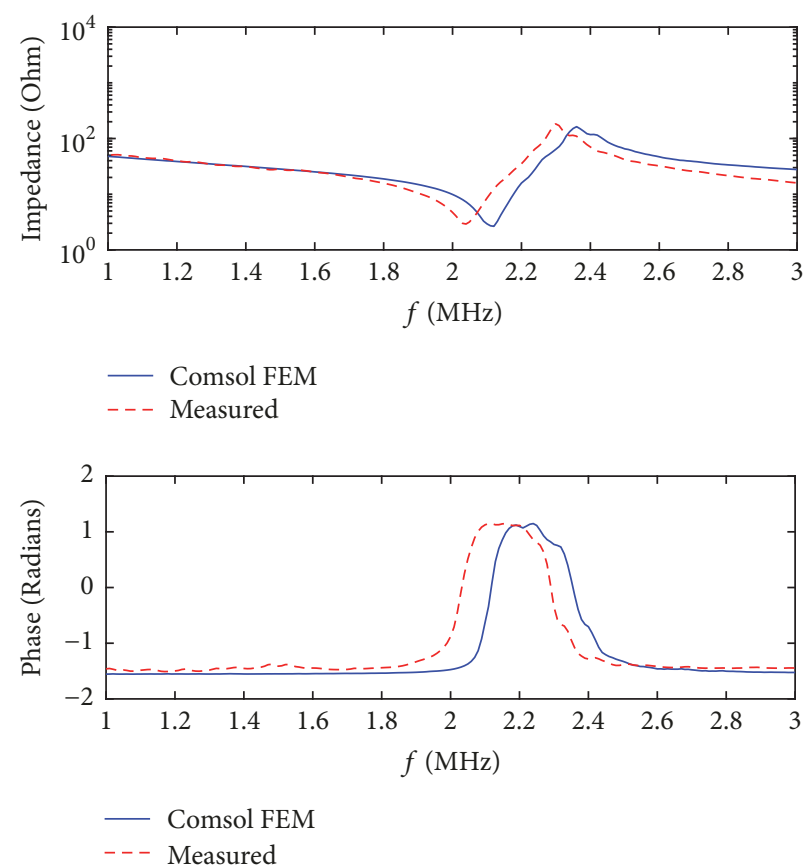

FIGURE 15: Equivalent input impedance of the channel through a cylindrical metal barrier. 


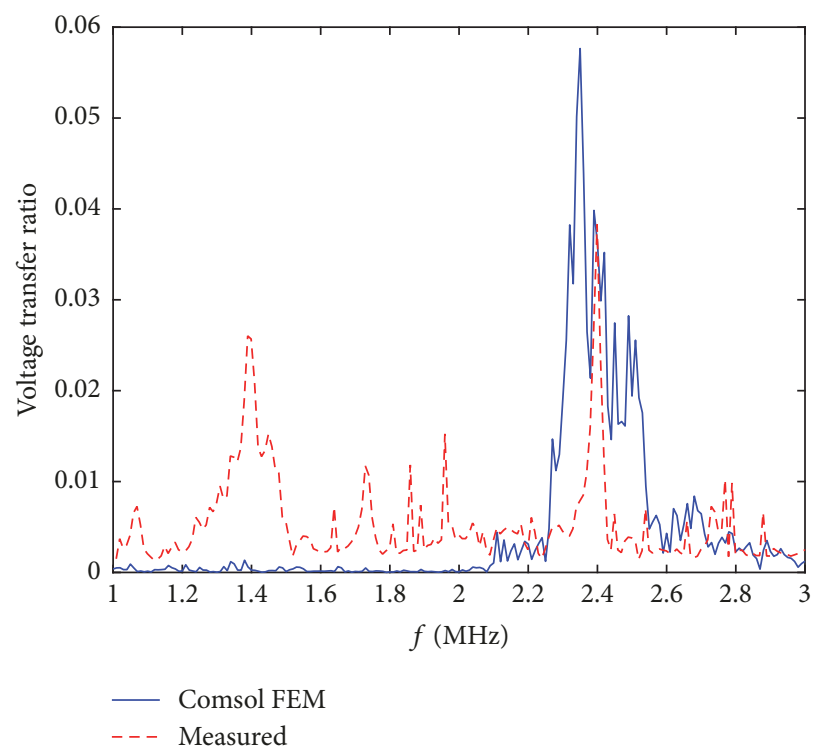

FIGURE 16: Output-to-input voltage transfer ratio of the channel through a cylindrical metal pipe.

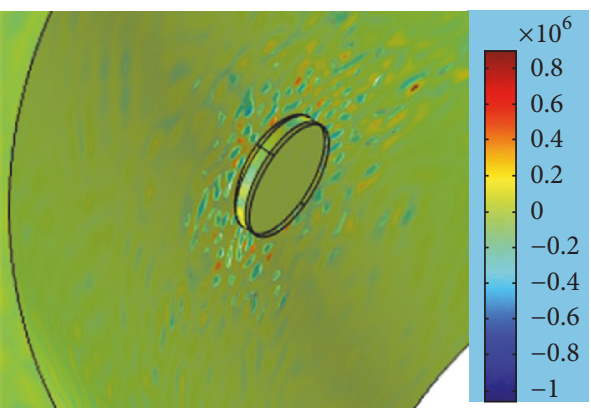

(a) $f=2.009 \mathrm{MHz}$

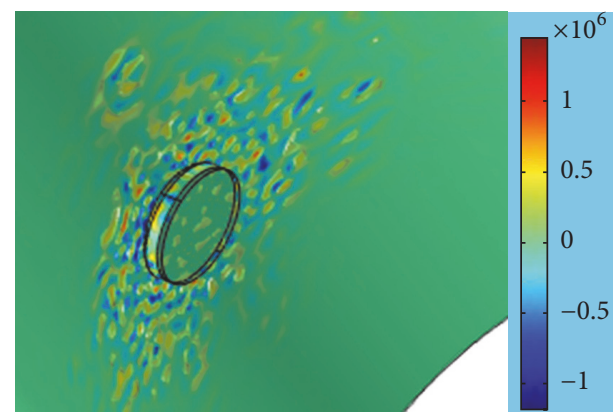

(b) $f=2.24 \mathrm{MHz}$

FIgURE 17: Acoustic pressure distributions in the cylindrical metal barrier from FEM.

element modeling method both can be applied to the optimal design of physical ultrasonic energy transmission systems through a planar barrier.

For the channel through a curved cylindrical metal pipe, characteristics including the input impedance and voltage transfer ratio have been simulated based on the three-dimensional finite element model, which are compared with experimental measurements. The simulated input impedance curve is well matched to that from measurement, verifying the effectiveness of the finite element modeling method. But both simulation and experimental results have shown that there are no multiple peaks and valleys in the input impedance characteristics and the voltage transfer ratio curves. This phenomenon is different from that occurring in the channel through a planar metal barrier. And the channel's voltage transfer ratio is relatively low, which implies that the energy transmission capability of the planar metal barrier is better than that of the cylindrical metal barrier under the same conditions. The main reason may be due to the fact that, for planar barriers, the effect of standing waves on the efficiency of wireless energy transmission is dominated, but for the cylindrical barrier, there are no standing waves formed and only the resonant phenomenon of the piezoelectric transducer exists.

\section{Conflicts of Interest}

The authors declare that there are no conflicts of interest regarding the publication of this paper.

\section{Acknowledgments}

The authors greatly appreciate the support provided by National Natural Science Foundation of China no. 51375485 and Natural Science Foundation of Hunan Province no. 2017JJ2300 for this work.

\section{References}

[1] Y. Hu, X. Zhang, J. Yang, and Q. Jiang, “Transmitting electric energy through a metal wall by acoustic waves using piezoelectric transducers," IEEE Transactions on Ultrasonics, Ferroelectrics and Frequency Control, vol. 50, no. 7, pp. 773-781, 2003. 
[2] Z. Yang, S. Guo, and J. Yang, "Transmitting electric energy through a closed elastic wall by acoustic waves and piezoelectric transducers," IEEE Transactions on Ultrasonics, Ferroelectrics and Frequency Control, vol. 55, no. 6, pp. 1380-1386, 2008.

[3] Z. Yang, J. Yang, and Y. Hu, "Energy trapping in power transmission through an elastic plate by finite piezoelectric transducers," IEEE Transactions on Ultrasonics, Ferroelectrics and Frequency Control, vol. 55, no. 11, pp. 2493-2501, 2008.

[4] S. Sherrit, M. Badescu, X. Bao, Y. Bar-Cohen, and Z. Chang, "Efficient electromechanical network models for wireless acoustic-electric feed-throughs," in Proceedings of the Smart Structures and Materials 2005 - Smart Sensor Technology and Measurement Systems, pp. 362-372, March 2005.

[5] Z. Chang, X. Bao, B. J. Doty et al., "Power loss consideration in wireless piezoelectric acoustic-electric power feedthru," in Proceedings of the Sensors and Smart Structures Technologies for Civil, Mechanical, and Aerospace Systems 2007, March 2007.

[6] S. Moss, P. McMahon, C. Konak et al., "Modelling and experimental validation of the acoustic electric feedthrough technique. , air vehicles division," Research Report DSTO-RR-0338, 2008.

[7] S. Moss, J. Skippen, M. Konak, I. Powlesland, and S. Galea, "Detachable acoustic electric feedthrough," in Proceedings of the Sensors and Smart Structures Technologies for Civil, Mechanical, and Aerospace Systems 2010, March 2010.

[8] K. R. Wilt, H. A. Scarton, S. Roa-Prada et al., "Finite element modeling and simulation of a two-transducer through-wall ultrasonic communication system," in Proceedings of the 2009 ASME International Mechanical Engineering Congress and Exposition, IMECE2009, pp. 579-589, November 2009.

[9] K. R. Wilt, T. J. Lawry, H. A. Scarton et al., "Mechanical design implications on power transfer through thick metallic barriers using piezoelectric transducers," in Proceedings of the ASME 2010 International Mechanical Engineering Congress and Exposition, IMECE 2010, pp. 173-182, November 2010.

[10] K. R. Wilt, T. J. Lawry, H. A. Scarton, and G. J. Saulnier, "One-dimensional pressure transfer models for acoustic-electric transmission channels," Journal of Sound and Vibration, vol. 352, Article ID 12466, pp. 158-173, 2015.

[11] Z. Yang, D. Zeng, H. Wang, C. Zhao, and J. Tan, "Harvesting ultrasonic energy using 1-3 piezoelectric composites," Smart Materials and Structures, vol. 24, no. 7, Article ID 075029, 2015.

[12] W. M. Leach, "Controlled-source analogous circuits and SPICE models for piezoelectric transducers," IEEE Transactions on Ultrasonics, Ferroelectrics and Frequency Control, vol. 41, no. 1, pp. 60-66, 1994.

[13] S. Roa-Prada, H. A. Scarton, G. J. Saulnier et al., "An ultrasonic through-wall communication (UTWC) system model," Journal of Vibration and Acoustics, vol. 135, no. 1, Article ID 011004, 2013. 


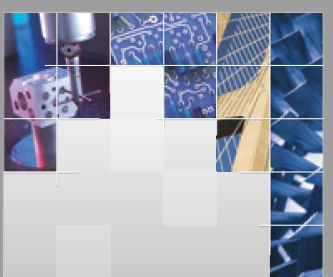

\section{Enfincering}
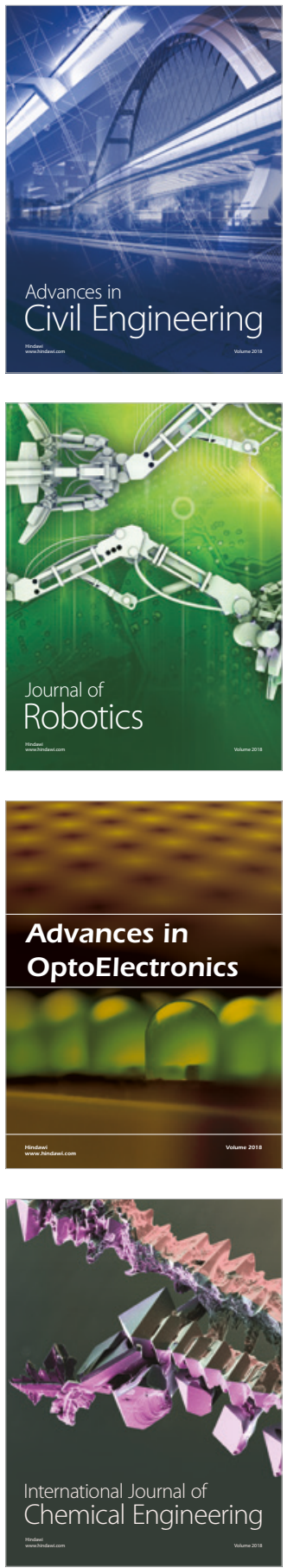

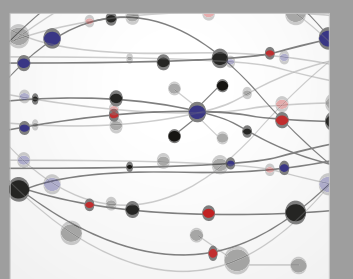

\section{Rotating \\ Machinery}

The Scientific World Journal

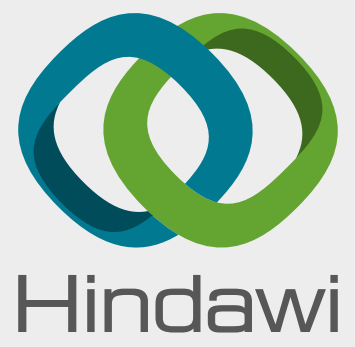

Submit your manuscripts at

www.hindawi.com
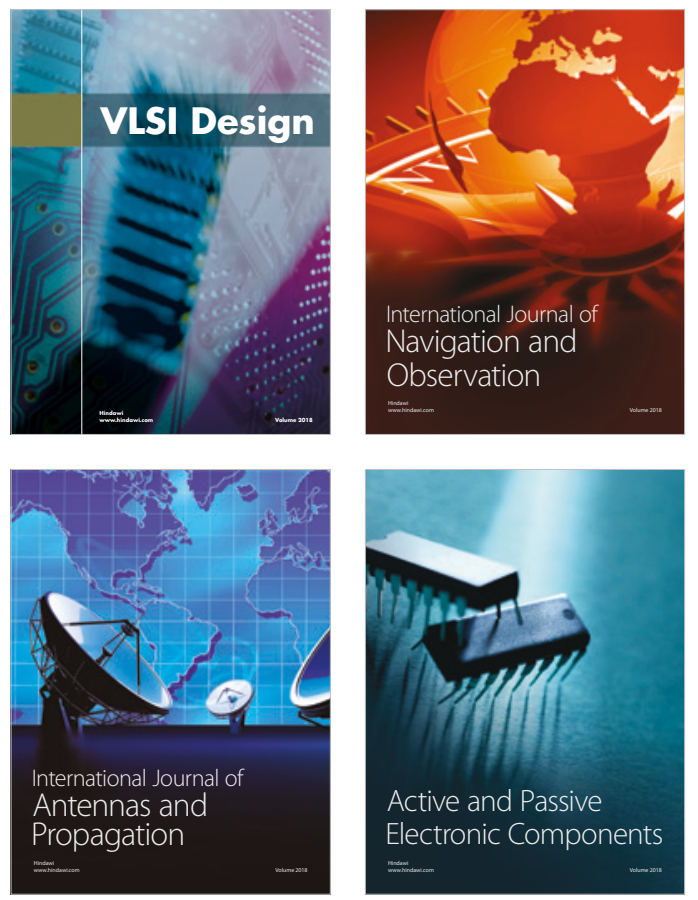
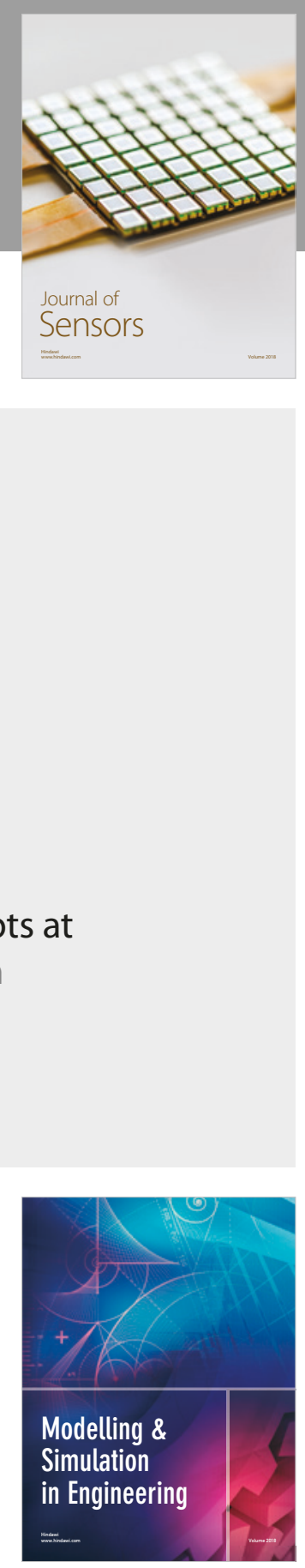

\section{Advances \\ Multimedia}
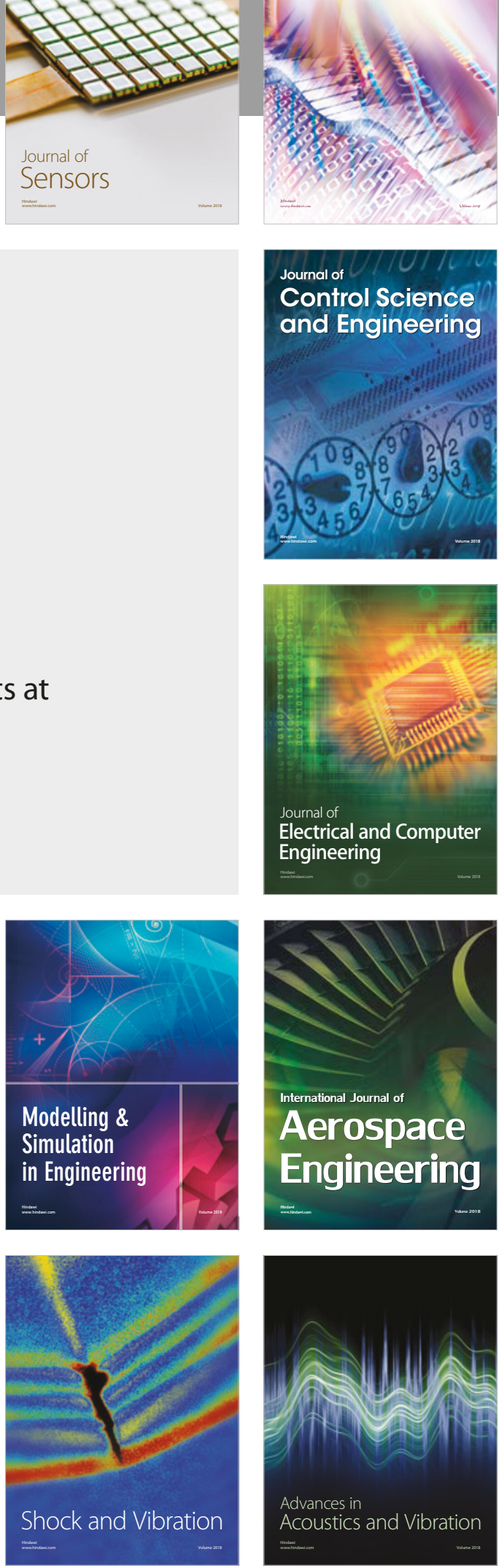